\title{
Accelerated hydrothermal aging of cycloaliphatic epoxy/graphene nanoparticle composites
}

\author{
J.M. Tomasi, I.D. Helman, W.A. Pisani, D.R. Klimek-McDonald, S. Chinkanjanarot, I. \\ Miskioglu, J.A. King, G.M. Odegard \\ Michigan Technological University \\ 1400 Townsend Drive \\ Houghton, MI 49931
}

\begin{abstract}
Cycloaliphatic epoxy (CE) resin systems are of primary interest in applications that require improved resistance to harsh conditions relative to other epoxy systems. Because other epoxy systems have demonstrated improved resistance to hydrothermal aging with the addition of carbon-based nanoparticle reinforcement, it is expected that the hydrothermal resistance of CE resins will likewise be improved with incorporation of nanoparticles. Therefore, the objective of this study is to determine the influence of graphene nanoparticles (GNP) on the hydrothermal aging resistance of CE resins. CE specimens are fabricated with varying levels of GNP and exposed to elevated temperatures and moisture levels for varying amounts of time up to 400 hours. The results from flexure and dynamical-mechanical testing indicate that the addition of GNP provides modest improvements in the stiffness and glass transition temperature for all aging levels, while the strength is improved for aging times below 400 hours.
\end{abstract}

KEYWORDS: Nanocomposites; Degradation; Hygrothermal; Weathering

\section{Introduction}

Cycloaliphatic epoxies (CE) are commonly used in structural applications requiring improved resistance to elevated temperatures and UV radiation relative to Bisphenol A (DGEBA) or Bisphenol F (DGEBF) Diglycidyl Ether epoxies. In one particular application, CE-based epoxy composites are used for structural reinforcement in aluminum conductor composite core (ACCC) power lines [1, 2]. ACCC lines must withstand harsh environmental conditions, such as long-term mechanical fatigue, temperature, moisture, UV, and high electric field exposure [36]. Thus, CE epoxy composites must be designed with optimal resistance to aging to prevent the catastrophic failure of ACCC power lines.

Graphene nanoparticles (GNPs) are known to increase the Young's modulus of epoxies [7-9] and carbon fiber/epoxy composites [10] when sufficiently dispersed, with or without chemical functionalization. These increases are known to originate from the increasing level of load transfer from the epoxy to the stiffer GNPs with increasing levels of GNP volume fraction. A limited number of studies have focused on examining the influence of graphene-based fillers on the aging characteristics of 
epoxies. Starkova et al. [11] demonstrated the influence of hydrothermal aging on the properties of DGEBA reinforced with thermally reduced graphene oxide (TRGO) particles. Prolongo et al. [12] examined the moisture uptake of GNP/DGEBA composites for various levels of GNP loading. To the authors' knowledge, no study has yet been performed on the effects of hydrothermal aging on the mechanical properties of GNP/CE nanocomposites.

The goal of this study is to determine the influence of GNPs on the mechanical properties of hydrothermally aged CEs. The following sections describe the materials, fabrication techniques, and test methods used to establish the dependency of CE mechanical properties on GNP loading and hydrothermal aging time. The results are compared to the results of similar studies found in the literature.

\section{Materials}

The CE-based epoxy used in this study consisted of a CE resin (3,4epoxycyclohexylmethyl-3,4-epoxycyclohexylcarboxlate) blended with relatively small amounts of DEGBA (specific blend ratio is proprietary with the supplier). This blend was cured by an anhydride (4, 7-methanoisobenzofuran-1, 3-dione-3a, 7, 7atetrahydromethyl). The chemical structures of these three molecules are shown in Figure 1. The curing mechanism is a chain reaction that starts with the opening of an anhydride ring that exposes a carboxyl group. The carboxyl group reacts with an epoxide group to form a chain with a new exposed hydroxyl group, which is used in a subsequent reaction with either an epoxide or anhydride group. These reactions proceed until there are no more epoxide groups available for reaction and the crosslinked system is a solid matrix.

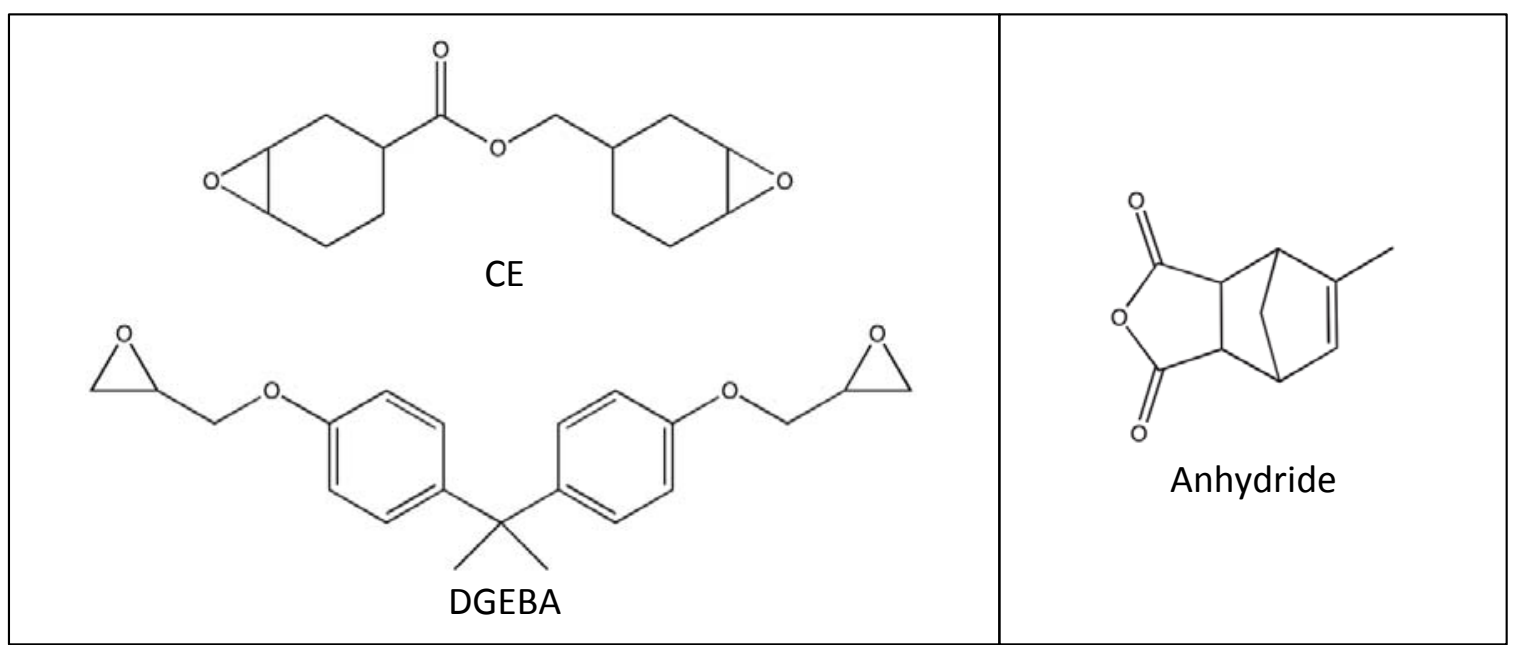

Figure 1 - Monomer molecules used for solid epoxy matrix

The GNPs used in this study were Asbury Carbon TC307. TC307 has an average particle diameter of $<1 \mu \mathrm{m}$, a surface area of $350 \mathrm{~m}^{2} / \mathrm{g}$, a density of $\sim 2 \mathrm{~g} / \mathrm{mL}$, and consist of $\sim 8$ layers of graphene as received. It was previously shown by the current 
research group in the study of Hadden et al [10] that the material preparation procedures described below are effective in reducing the layers from $\sim 8$ to $\sim 4$ in the final composite material. It was further shown by Hadden et al. [10] that the number of layers, thus the level of dispersion, plays a critical role in the overall performance of composite materials. Although an exact laboratory-based determination of the average number of graphene layers is very difficult, Hadden et al [10] demonstrated that a combination of modeling and experiment could be used to efficiently establish the effect of graphene layers on overall composite properties.

\section{Specimen fabrication}

For the neat epoxy material, a multi-part mold was coated with Mann Ease Release 300 , then assembled and pre-heated in a vacuum oven at $100^{\circ} \mathrm{C}$. The appropriate amounts of resin and hardener (proprietary mixing ratio) were weighed into a beaker. The beaker was placed under a Ross High Shear Mixer (HSM-100 LSKI) with 2" dispersion blade. The mixture was mixed at $1000 \mathrm{rpm}$ for $10 \mathrm{~min}$, until the two parts were thoroughly mixed. The mixture was then degased at $100{ }^{\circ} \mathrm{C}$ and $29 \mathrm{in} \mathrm{Hg}$, alternating vacuum with atmospheric pressure to avoid overflow, until no more bubbles appeared. The mixture was then poured into the pre-heated mold and degased once more. The curing cycle used was $100^{\circ} \mathrm{C}$ for 1 hour, then heated to 200 ${ }^{\circ} \mathrm{C}$ at $2{ }^{\circ} \mathrm{C} / \mathrm{min}$ then held at $200{ }^{\circ} \mathrm{C}$ for two hours. The oven was then turned off and the cured epoxy was allowed to cool in the oven to room temperature at a $\sim 1$ ${ }^{\circ} \mathrm{C} / \mathrm{min}$ cooling rate.

For the 2 and 4 wt\% GNP material systems, a multi-part mold was coated with Mann Ease Release 300 , then assembled and pre-heated in a vacuum oven at $100{ }^{\circ} \mathrm{C}$. The amount of GNP needed was weighed into a beaker, along with the corresponding amount of hardener. The beaker was then placed under a Ross High Shear Mixer (HSM-100 LSKI) with 2" dispersion blade. The mixture was mixed at $3000 \mathrm{rpm}$ for 1 hour, then the mixture was placed in a Branson Sonicator CPX2800H operating at $40 \mathrm{kHz}$ and sonicated for 1 hour. The corresponding amount of resin was added to the mixture, then mixed at $1000 \mathrm{rpm}$ for $10 \mathrm{~min}$. The mixture was then degased at $100{ }^{\circ} \mathrm{C}$ and $29 \mathrm{in} \mathrm{Hg}$, alternating vacuum with atmospheric pressure to avoid overflow, until no more bubbles appeared. The mixture was poured into the preheated mold and degased once more. The curing cycle used was $100^{\circ} \mathrm{C}$ for 1 hour, then heated to $200^{\circ} \mathrm{C}$ at $2{ }^{\circ} \mathrm{C} / \mathrm{min}$, then held at $200^{\circ} \mathrm{C}$ for two hours. The oven was turned off and the cured epoxy was allowed to cool in the oven to room temperature, $\sim 1^{\circ} \mathrm{C} / \mathrm{min}$ cooling rate.

From the fabricated plaques a series of 3-point bend and dynamical mechanical analysis (DMA) specimens were machined. The 3-point bend specimens had dimensions of $127 \times 12.7 \times 3.2 \mathrm{~mm}$ per ASTM D790, and the DMA single-cantilever beam specimens had dimensions of $35 \times 12.7 \times 3.2 \mathrm{~mm}$. A total of 203 -point bend and 20 DMA specimens were machined for each of the three materials systems: neat, 2 $\mathrm{wt} \%$, and $4 \mathrm{wt} \% \mathrm{GNP}$. Thus, a total of 603 -point bend and 60 DMA specimens were 
prepared. Figure 2 shows a SEM image of a fracture surface of the composite material.

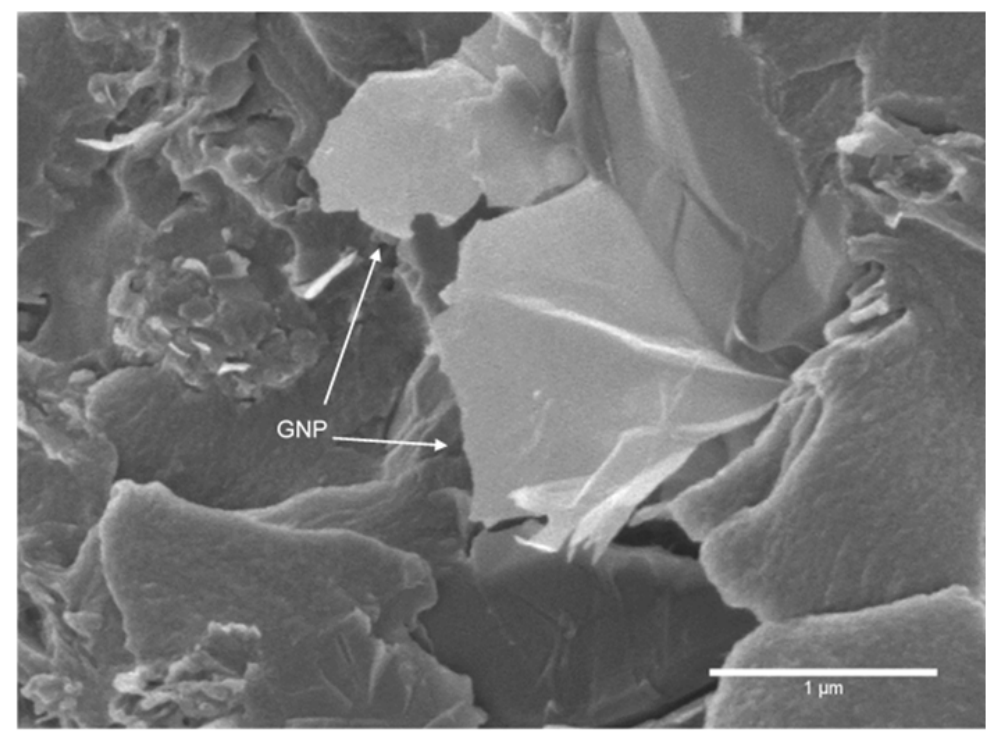

Figure 2 - Scanning Electron Microscopy image of the GNP/epoxy composite

\section{Testing Procedure}

A QUV Accelerated Weathering Testing Machine was used to condition the specimens under controlled temperature $\left(50^{\circ} \mathrm{C}\right)$ and humidity conditions $(100 \%$ relative humidity). Both 3-point bend and DMA specimens were exposed to these conditions for $0,50,100,200$, and 400 hours. Before aging all specimens were conditioned at $50 \%$ relative humidity and $23^{\circ} \mathrm{C}$ for two days.

During the conditioning, mass measurements were made on the DMA specimens at regular intervals to monitor the moisture uptake. For each mass measurement, the specimens were quickly removed from the weathering chamber and sealed in a moisture barrier bag to cool to room temperature (10 minutes). After cooling, the specimens were removed from the bags, excess surface moisture removed, and mass measurements performed. The specimens were returned to the weathering chamber immediately after measuring.

For both the 3-point bend and DMA specimens for each material system (neat, 2 $\mathrm{wt} \%, 4 \mathrm{wt} \% \mathrm{GNP}$ ), 4 specimens were left unaged, 4 specimens aged for 50 hours, 4 aged for 100 hours, 4 aged for 200 hours, and 4 were aged for 400 hours. After aging was complete, each specimen was immediately removed from the chamber and the excess surface moisture removed. The specimens were promptly sealed in moisture barrier bags and allowed to cool to room temperature before testing.

The 3-point bend tests were performed per the specifications of ASTM D790 with a crosshead rate of $5.3 \mathrm{~mm} / \mathrm{min}$ on an Instru-Met Sintech screw-driven mechanical 
testing machine with an LVDT to record specimen deflection. The flexural modulus and flexural strength values were calculated based on the load-deflection data using the equations outlined in ASTM D790.

The DMA tests were performed on a TA Instruments Q800 DMA using the single cantilever clamp fixture. An oscillating amplitude of $30 \mu \mathrm{m}$ was applied to the specimen, with an initial temperature of $50^{\circ} \mathrm{C}$ with a soak time of 5 minutes, a temperature ramp rate of $3^{\circ} \mathrm{C} / \mathrm{min}$, and a final temperature of $290^{\circ} \mathrm{C}$ with a hold time of 5 minutes. The frequency was held at $1 \mathrm{~Hz}$ for the entire duration of the test. Once the test was completed, the data was analyzed to determine the tan delta values for each specimen. The glass transition temperature was assumed to correspond to the peak of the tan delta values for each material system and aging level.

\section{Results and Discussion}

Figure 3 shows the mass gain of the nanocomposite over the 400 hours of hydrothermal exposure. The error bars represent a standard deviation over the multiple specimens measured for each exposure time interval. From the figure it is clear that during the first 100 hours the neat resin specimen absorbed water more quickly than the specimens with GNP content. The likely cause of this behavior is the increase in moisture path diffusion length in the presence of hydrophobic GNP. After 100 hours the specimens started to approach saturation, and the moisture content of all three material systems stabilized around $2 \%$. For the composite systems these results are similar to those of Starkova et al. [11] and Prolongo et al. [12]. The equilibrium water absorption level in the neat epoxy is consistent with that observed by Prolongo et al. [12] and less than half of the amount reported by Starkova et al. [11]. 


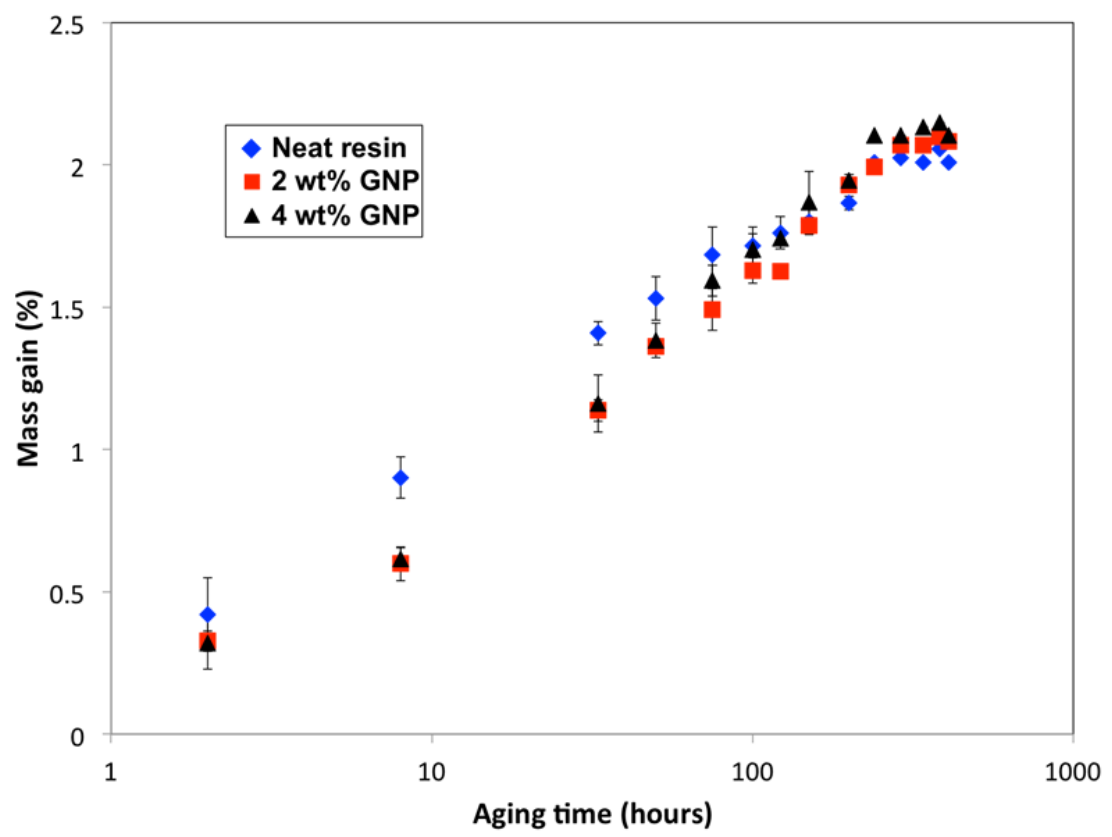

Figure 3 - Water mass gain for GNP/epoxy nanocomposites

Figure 4 shows the flexural modulus for all three material systems over the entire exposure time. The error bars represent a standard deviation over the multiple specimens tested for each material at each aging time. As is observed in many unaged epoxy nanocomposite systems [13-20], the increasing levels of nanoreinforcement resulted in increasing flexural modulus (stiffness) values for all exposure times, which indicates that the load transfer capability of the system remains effective throughout the aging process for sub-failure stress levels. For 50 and 100 hours of exposure, no significant change in the flexural modulus was observed for the materials relative to the unaged state. After 200 hours of exposure, a very modest decrease of only a few percent in the flexural modulus was observed for the neat resin and $2 \mathrm{wt} \%$ GNP materials. This result is consistent with Starkova et al. [11], who demonstrated a slight decrease in storage modulus with hydrothermally aged neat epoxy and graphene/epoxy composites ( $0.3 \mathrm{wt} \% \mathrm{TRGO}$ ) tested near room temperature. It is also apparent from Figure 4 that within the error bars, the $4 \mathrm{wt} \%$ GNP material appears to have a constant modulus over the full aging time. The observed change in modulus at 200 hours of exposure in the neat resin and $2 \mathrm{wt} \%$ GNP material could be due to plasticization with increasing moisture content, which is partially mitigated with higher levels of GNP content. 


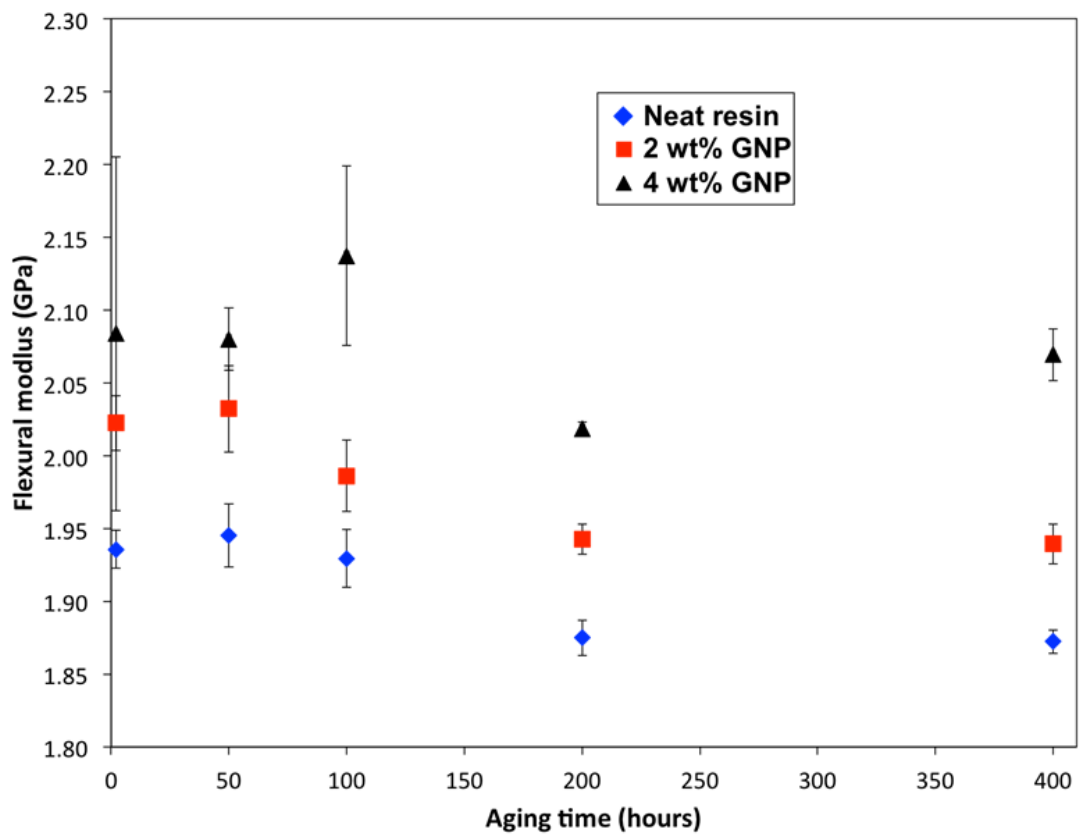

Figure 4 - Flexural modulus for GNP/epoxy nanocomposites

The flexural strength of each of the material systems for the entire exposure time is shown in Figure 5. There is a small increase in the material strength with increasing GNP loading for the complete set of aging times, which is typically observed in other epoxy nanocomposite systems $[17,18,21]$. There is no clear change in the flexural strength for all three materials up to 200 hours of exposure. At 400 hours, there is a few percent drop in strength for the $2 \mathrm{wt} \%$ and $4 \mathrm{wt} \%$ GNP materials, and no change for the neat resin. The subtle decrease in the strength at 400 hours could be due to degradation of the epoxy/GNP interface region in the nanocomposites that affects the ultimate failure of the interface but not the load transfer at lower loads (per the discussion above). 


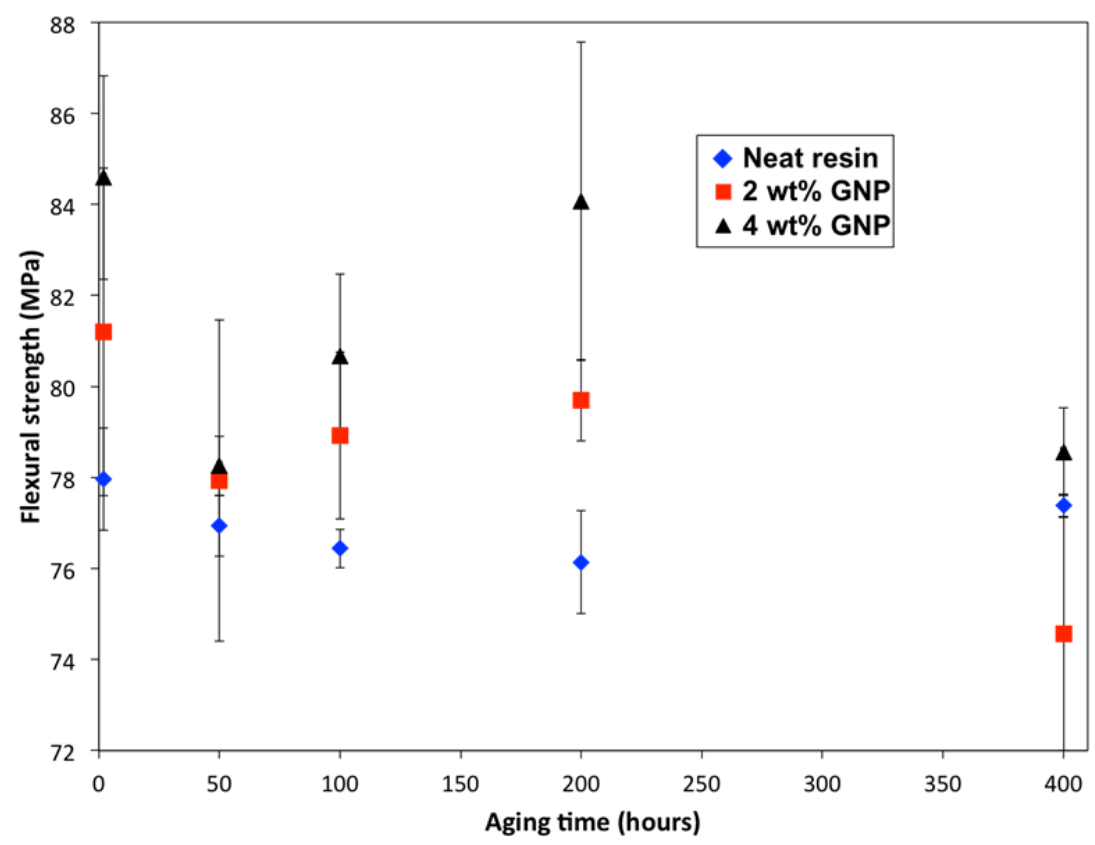

Figure 5 - Flexural strength for GNP/epoxy nanocomposites

The glass transition temperatures measured in the DMA tests are shown in Figure 6. The error bars represent a standard deviation over the multiple specimens measured for each exposure time interval (some error bars are hidden by the data points). The data demonstrates about a 1-2\% drop in the glass transition temperature for the three material systems with the first increment of aging. Thereafter, the glass transition temperature remains relatively constant for higher levels of aging. Starkova et al [11] observed a much more drastic drop in glass transition of neat DEGBA resin and DEGBA/TRGO composites with aging. The data in Figure 6 suggests that GNP loading does not strongly affect the glass transition in the unaged material (similar to trends observed in other epoxy nanocomposites [11, $13,19,22])$. However, for nearly all of the aged specimens there appears to be an increase in glass transition with addition of GNP into the epoxy. Thus, it appears that the epoxy/GNP interface still can retain load transfer capability during the aging process despite the effects of moisture plasticity on the epoxy resin. 


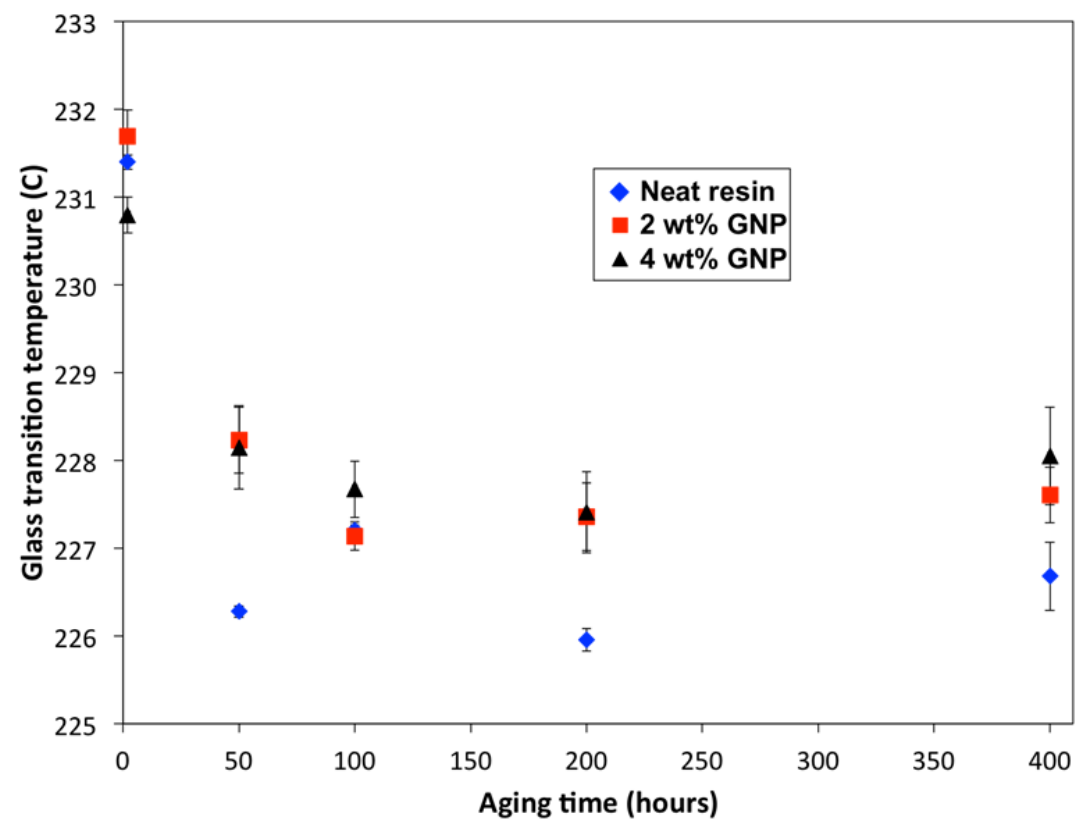

Figure 6 - Glass transition temperature for GNP/epoxy nanocomposites

\section{Conclusions}

The results of this study indicate that GNP reinforcement provides increases in the magnitude of stiffness regardless of the aging level and increases in material strength for aging times less than 400 hours. The results also suggest that GNP reinforcement provides increases in the glass transition temperature relative to the neat epoxy for hydrothermally aged specimens. However, despite these improvements, the composite material strengths were compromised at 400 hours and the overall magnitude of glass transition of the composite and neat resin materials was reduced relative to the unaged state. Therefore, it follows that the detrimental effects of epoxy moisture plasticity are partially mitigated by the load transfer capability of GNP, except in the case of the 400 hour aging time, where the composite strength drops and the neat resin strength remains unaffected.

It is also important to note that the neat $\mathrm{CE}$ resin shows a remarkable resistance to hydrothermal aging relative to the DEGBA resin reported by Starkova et al [11]. While the $\mathrm{CE}$ resin flexural modulus dropped about $4 \%$ between the unaged and 400 hour aging conditions, the DEGBA resin storage modulus dropped about $19 \%$ at $30^{\circ} \mathrm{C}$. Also, while the glass transition temperature of the $\mathrm{CE}$ resin dropped $2 \%$ after aging, that of the DEGBA resin dropped 61\%. Therefore, regardless of the GNP reinforcement loading level, the CE resin performs better under hydrothermal aging conditions than the DEGBA resin. 


\section{Acknowledgements}

This research was funded by NASA under the Aeronautical Sciences Program (Grant NNX11A072A), the NSF I/UCRC on Novel High Voltage/Temperature Materials and Structures (Grant IIP-1362040), the Richard and Elizabeth Henes endowment at Michigan Technological University, and the Department of Mechanical Engineering Engineering Mechanics at Michigan Technological University. The authors thank Asbury Carbon for donating graphene nanoplatelets. The authors would also like to thank the following undergraduate students for their assistance on this project: Chris Blevins, Bryan Cammin, Madison Diehl, Shalina Gupta, Gabe Rupright, and Garrett Unsworth.

\section{References}

1. Middleton, J., B. Burks, T. Wells, A. M. Setters, I. Jasiuk, M. Kumosa. 2013. "The effect of ozone and high temperature on polymer degradation in polymer core composite conductors". Polymer Degradation and Stability. 98(11):2282-2290.

2. Middleton, J., B. Burks, T. Wells, A. M. Setters, I. Jasiuk, P. Predecki, et al. 2013. "The effect of ozone on polymer degradation in Polymer Core Composite Conductors". Polymer Degradation and Stability. 98(1):436-445.

3. Burks, B., J. Middleton, M. Kumosa. 2012. "Micromechanics modeling of fatigue failure mechanisms in a hybrid polymer matrix composite". Composites Science and Technology. 72(15):1863-1869.

4. $\quad$ Burks, B., M. Kumosa. 2012. "The effects of atmospheric aging on a hybrid polymer matrix composite". Composites Science and Technology. 72(15):1803-1811.

5. Burks, B., D. Armentrout, M. Kumosa. 2011. "Characterization of the fatigue properties of a hybrid composite utilized in high voltage electric transmission". Composites Part a-Applied Science and Manufacturing. 42(9):1138-1147.

6. Burks, B., J. Middleton, D. Armentrout, M. Kumosa. 2010. "Effect of excessive bending on residual tensile strength of hybrid composite rods". Composites Science and Technology. 70(10):1490-1496.

7. King, J. A., D. R. Klimek, I. Miskioglu, G. M. Odegard. 2015. "Mechanical properties of graphene nanoplatelet/epoxy composites". Journal of Composite Materials. 49(6):659-668.

8. King, J. A., D. R. Klimek, I. Miskioglu, G. M. Odegard. 2013. "Mechanical properties of graphene nanoplatelet/epoxy composites". Journal of Applied Polymer Science. 128(6):4217-4223.

9. Potts, J. R., D. R. Dreyer, C. W. Bielawski, R. S. Ruoff. 2011. "Graphene-based polymer nanocomposites". Polymer. 52(1):5-25.

10. Hadden, C. M., D. R. Klimek-McDonald, E. J. Pineda, J. A. King, A. M. Reichanadter, I. Miskioglu, et al. 2015. "Mechanical properties of graphene nanoplatelet/carbon fiber/epoxy hybrid composites: Multiscale modeling and experiments". Carbon. 95:100-112. 
11. Starkova, O., S. Chandrasekaran, L. A. S. A. Prado, F. Tolle, R. Mulhaupt, K. Schulte. 2013. "Hydrothermally resistant thermally reduced graphene oxide and multi-wall carbon nanotube based epoxy nanocomposites". Polymer Degradation and Stability. 98(2):519-526.

12. Prolongo, S. G., R. Moriche, A. Jimenez-Suarez, M. Sanchez, A. Urena. 2014. "Advantages and disadvantages of the addition of graphene nanoplatelets to epoxy resins". European Polymer Journal. 61:206-214.

13. Etika, K. C., L. Liu, L. A. Hess, J. C. Grunlan. 2009. "The influence of synergistic stabilization of carbon black and clay on the electrical and mechanical properties of epoxy composites". Carbon. 47(13):3128-3136.

14. Kosmidou, T. V., A. S. Vatalis, C. G. Delides, E. Logakis, P. Pissis, G. C. Papanicolaou. 2008. "Structural, mechanical and electrical characterization of epoxy-amine/carbon black nanocomposites". Express Polymer Letters. 2(5):364-372.

15. Sun, Y. Y., Z. Q. Zhang, K. S. Moon, C. P. Wong. 2004. "Glass transition and relaxation behavior of epoxy nanocomposites". Journal of Polymer Science Part B-Polymer Physics. 42(21):3849-3858.

16. Ratna, D., N. R. Manoj, R. Varley, R. K. S. Raman, G. P. Simon. 2003. "Clayreinforced epoxy nanocomposites". Polymer International. 52(9):1403-1407.

17. Wang, X., W. Y. Xing, P. Zhang, L. Song, H. Y. Yang, Y. Hu. 2012. "Covalent functionalization of graphene with organosilane and its use as a reinforcement in epoxy composites". Composites Science and Technology. 72(6):737-743.

18. Raza, M. A., A. Westwood, C. Stirling, R. Brydson, N. Hondow. 2012. "Effect of nanosized carbon black on the morphology, transport, and mechanical properties of rubbery epoxy and silicone composites". Journal of Applied Polymer Science. 126(2):641-652.

19. Dittanet, P., R. A. Pearson. 2012. "Effect of silica nanoparticle size on toughening mechanisms of filled epoxy". Polymer. 53(9):1890-1905.

20. Zaman, I., T. T. Phan, H. C. Kuan, Q. S. Meng, L. T. B. La, L. Luong, et al. 2011. "Epoxy/graphene platelets nanocomposites with two levels of interface strength". Polymer. 52(7):1603-1611.

21. Ma, A. J., W. X. Chen, Y. G. Hou, G. Zhang. 2010. "Dispersion, Mechanical and Thermal Properties of Epoxy Resin Composites Filled with the Nanometer Carbon Black". Polymer-Plastics Technology and Engineering. 49(9):916-920.

22. Han, J. T., K. Cho. 2006. "Nanoparticle-induced enhancement in fracture toughness of highly loaded epoxy composites over a wide temperature range". Journal of Materials Science. 41(13):4239-4245. 